\title{
Multiple Epitope Tagging of Expressed Proteins for Enhanced Detection
}

\author{
Ron Hernan, Ken Heuermann and Bill Brizzard \\ Sigma, St. Louis, MO, USA
}

BioTechniques 28:789-793 (April 2000)

\begin{abstract}
Three FLAG epitopes have been incorporated into the mammalian expression vector pCMV-5 to create a transient expression vector, p3XFLAG-CMV-7. The vector was designed to express FLAG fusion proteins that can be detected at tenfold lower expression levels than the current FLAG fusion protein expression system. The usefulness of this expression and detection system was demonstrated by expression of bacterial alkaline phosphatase in COS-7 cells. In addition, $3 X F L A G$ bacterial alkaline phosphatase was expressed in Escherichia coli, purified on anti-FLAG M2 affinity gel, and detection of $500 \mathrm{pg}$ of purified protein by Western blot analysis is demonstrated.
\end{abstract}

\section{INTRODUCTION}

Epitope tagging has become a powerful tool for the detection and purification of expressed proteins. This methodology has been used for protein localization, immunoprecipitation and protein-protein interaction. Many types of tags have been used, with c-myc and FLAG tags being two of the most popular epitopes used (5). Generally, these epitopes are fused to the $\mathrm{N}$ - or C-terminus of the expressed protein, which makes them more accessible to the antibody for detection and less likely to cause severe structural or functional perturbations.

The original FLAG sequence, Asp-Tyr-Lys-Asp-Asp-AspAsp-Lys, is recognized by two monoclonal antibodies, M1 and M2 $(6,14)$. In addition, the FLAG sequence with an initiator methionine attached is recognized by the M2 antibody and a third antibody, M5 (2). The last five amino acids of the FLAG sequence are the recognition site for the protease enterokinase, which allows the removal of the FLAG epitope.

The FLAG epitope has been used in expression systems for detection and purification of heterologous proteins in Escherichia coli (3), Saccharomyces cerevisiae $(10,14)$ Drosophila (20), baculovirus $(4,15)$ and mammalian systems (13, 16). For mammalian expression systems, expression levels are low, and the effective detection of expressed foreign proteins using established methods can be difficult. We describe a mammalian expression plasmid containing multiple FLAG epitopes in tandem, p3XFLAG CMV-7, designed for intracellular expression with increased sensitivity of detection. This vector contains the cytomegalovirus (CMV) promoter (19) and simian virus 40 (SV40) origin of replication for efficient expression in COS-7 cells (12). Moreover, the detection of triple FLAG-tagged BAP that was expressed and purified from $E$. coli was compared to single FLAG-tagged BAP.

\section{MATERIALS AND METHODS}

All materials were supplied by Sigma (St. Louis, MO, USA) unless otherwise stated.

\section{p3XFLAG-CMV-7 Construction}

p3XFLAG-CMV-7 was constructed from the mammalian expression vector, pCMV-5 (1). The triple FLAG sequence was constructed from two pairs of complementary oligonucleotides. The first pair of oligonucleotides was synthesized as follows: 5'-GAAGAATTCACCATGGACTACAAAGACCATGACGGTGATTATAAAGATCATGAT-3' and 5'-ATCATGATCTTTATAATCACCGTCATGGTCTTTGTAGTCCAT GGTGAATTCTTC-3'. The second pair was synthesized with the following sequence: 5'-GAAGATATCGATTACAAGGATGACGATGACAAGCTTGGG-3' and 5'-CCCAAGCTTGTCATCGTCATCCTTGTAATCGATATCTTC-3'. The first pair of oligonucleotides were annealed together and digested with EcoRI and EcoRV. The second pair of oligonucleotides were annealed together and digested with EcoRV and HindIII. The two pairs of digested nucleotide cassettes were ligated into CMV-5, which had been double-digested with EcoRI and HindIII. The nucleotide sequence was verified by automated DNA sequencing.

\section{Construction of pFLAG-CMV7-BAP}

A modified version of the E. coli phoA gene (7) (for which 
the leader sequence and the N-terminal four amino acids of the mature enzyme were deleted) was subcloned into the vector p3XFLAG-CMV-7 to express BAP. The modified sequence was cut from pFLAG-ATS-BAP by double digestion with HindIII and BglII. The fragment was then cloned into p3XFLAG CMV-7, which had been double-digested with HindIII and BamHI to generate p3XFLAG-CMV-7-BAP. The nucleotide sequence at the $\mathrm{N}$-terminus of the phoA coding region was verified by automated DNA sequencing.

\section{Triple FLAG-ATS-BAP Construction}

Two oligonucleotides encoding the sense and antisense strand for the triple flag sequence were synthesized (sequences not shown), 5'-phosphorylated with T4 polynucleotide kinase and annealed together to generate a cassette with an NdeI and a HindIII restriction enzyme overhang. The pFLAG-ATS-BAP was digested with NdeI and HindIII, and the vector was purified by gel electrophoresis. The annealed cassette was ligated to the double-digested pFLAG-ATS-BAP vector with T4 DNA ligase, and the reaction was carried out overnight at $16^{\circ} \mathrm{C}$ for $16 \mathrm{~h}$. The ligation was enriched by di- gestion with NruI to linearize the parent vector that was not ligated with the triple FLAG sequence, and then transformed into $E$. coli $\mathrm{DH} 5 \alpha$. Clones were isolated, and the nucleotide sequence was verified by automated DNA sequencing.

\section{Bacterial Expression and Protein Purification}

E. coli BL21 (DE3) cells were transformed with the expression plasmid containing the triple FLAG BAP construct. Cells were grown in Terrific broth containing $100 \mu \mathrm{g} / \mathrm{mL}$ ampicillin at $37^{\circ} \mathrm{C}$ with agitation. The culture was grown to an $\mathrm{A}_{600}=4.0$ and then induced with IPTG at a final concentration of $1 \mathrm{mM}$. The cell culture was grown for an additional $3 \mathrm{~h}$ at $37^{\circ} \mathrm{C}$ and then harvested by centrifugation. The cell pellet was resuspended in $50 \mathrm{mM}$ Tris- $\mathrm{HCl}, \mathrm{pH} 8.0$, and the cells were disrupted by sonication. Then, the cellular debris was removed by centrifugation. BAP was affinity-purified on M2 antibody-conjugated sepharose $4 \mathrm{~B}$ resin equilibrated with 50 $\mathrm{mM}$ Tris- $\mathrm{HCl}, \mathrm{pH} 8.0,150 \mathrm{mM} \mathrm{NaCl}$ (TBS). The resin was washed with 20 bed volumes of TBS, and then the triple FLAG BAP was eluted with five column volumes of $0.1 \mathrm{M}$ Glycine, $\mathrm{pH}$ 3.5. The eluted protein was pooled and adjusted

A

ACC ATG GAC TAC AAA GAC CAT GAC GGT GAT TAT AAA GAT CAT GAT ATC GAT TAC TAG CTS ATG TTT CTG GTA CTG CCA CTA ATA TTT CPA GTA CTA TAG CTA ATE Met Asp Tyr Lys Asp His Asp Gly Asp Tyr Lys Asp His Asp Ile Asp Tyr

Hind III Pst I Sal I Xba I BamH I Sma I AAG GAT GAC GAT GAC AAG CTT GCA TGC CTG CAG QTC GAC ICT AGA GGA TCG qGG G TAC CTA CTG CTA CTG ITC GAA CGT ACG GAC GIC CAG CIG AGA TQT CCT AGG dCC C Lys Asp Asp Asp Asp Lys Leu Ala Cys Leu Gln Val Asp Ser Arg Gly Ser Arg

B

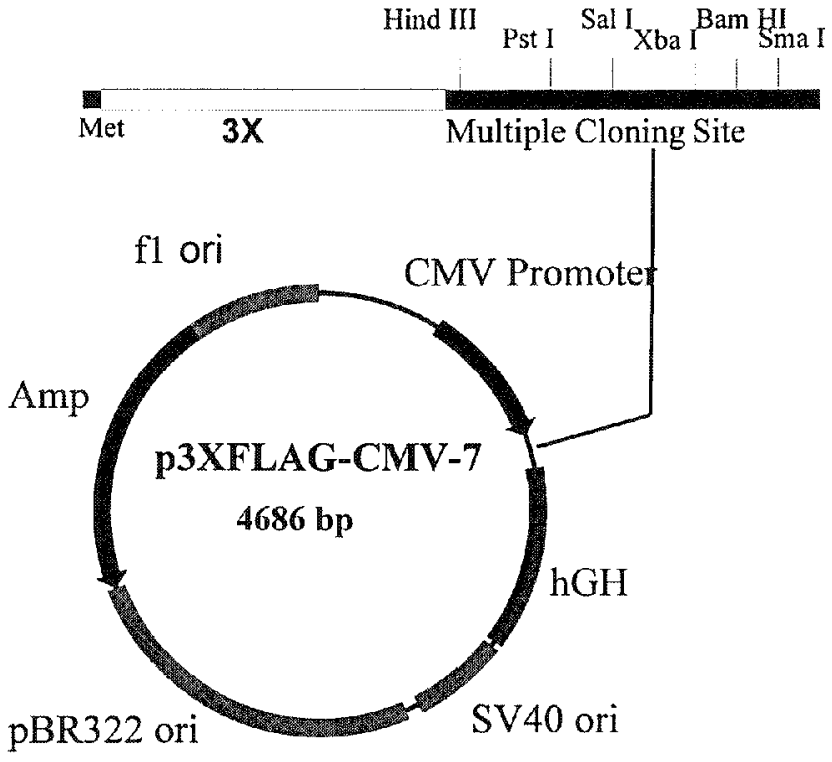

Figure 1. p3XFLAG-CMV-7 expression vector. (A) DNA and protein sequence of 3XFLAG-CMV-7 multiple cloning site and FLAG sequences. (B) Plasmid map of the p3XFLAG-CMV-7 showing the CMV promoter, human growth hormone transcription termination and polyadenylation site, SV40 origin of replication, $\mathrm{Col} \mathrm{E} 1$ origin of replication and $\beta$-lactamase gene. 
to $\mathrm{pH} 7.5$ with $1 \mathrm{M}$ Tris- $\mathrm{HCl}$, pH 8.0. Protein content was determined by the Bradford method and by absorbance using $\varepsilon_{280}=0.7 \mathrm{~mL} / \mathrm{mg}$; both methods yielded the same value.

\section{Western Blot}

Purified 3XFLAG-BAP and N-BAP were diluted with to $2 \times$ Laemlli buffer (9), boiled for $5 \mathrm{~min}$ and then placed on ice. Samples were resolved on a $15 \%$ SDS-PAGE using the method of Laemlli (9) and then transferred to nitrocellulose membranes. The membrane was blocked with PBS containing 3\% non-fat dry milk for $1 \mathrm{~h}$ and then rinsed $3 \times$ in TBS, $0.05 \%$ Tween ${ }^{\circledR} 20$ (TBS-T). The membrane was incubated with M2 antibody at a final concentration of $10 \mu \mathrm{g} / \mathrm{mL}$ for 30 min in TBS-T and then rinsed $3 \times$ in TBS-T. The membrane was then incubated for $30 \mathrm{~min}$ with a goat anti-rabbit $\mathrm{IgG}$ (whole molecule) horseradish peroxidase (HRP) conjugate diluted 1:10000 in TBS-T, then rinsed $3 \times$ in TBS-T. The FLAG-tagged proteins were detected with the HRP conjugate and visualized by chemilumenescent detection using an ECL $^{\mathrm{TM}}$ kit (Amersham Pharmacia Biotech, Piscataway, NJ, USA) with exposures from 1-30 min on Kodak X-Omat ${ }^{\circledR}$ MR film (Eastman Kodak, Rochester, NY, USA).

\section{Transfection of COS-7 Cells with p3XFLAG-CMV-7-BAP}

COS-7 cells were cultured on $35 \mathrm{~mm}^{2}$ plates in DMEM containing $10 \%$ fetal bovine serum, $4 \mathrm{mM}$ L-glutamine, 5

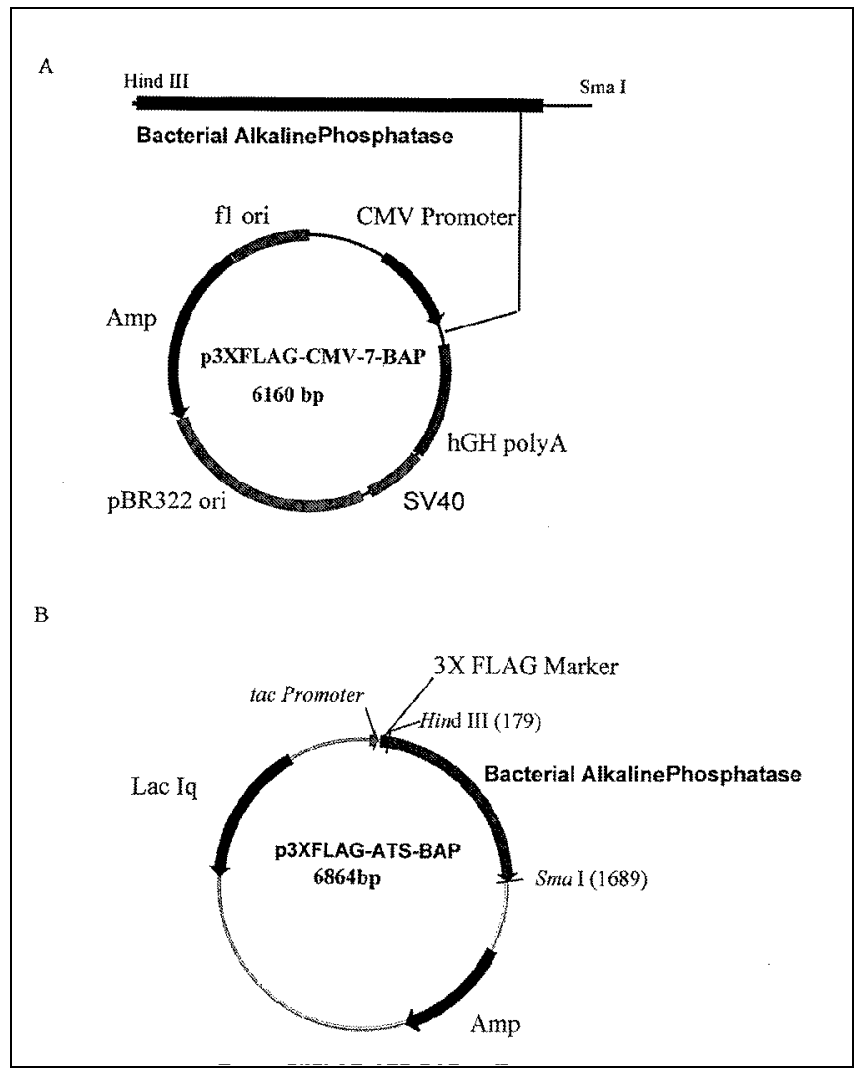

Figure 2. Expression vectors p3XFLAG-CMV-7-BAP and p3XFLAGATS-BAP. (A) Vector map of p3XFLAG-CMV-7-BAP showing insertion of the phoA coding region into p3XFLAG-CMV-7. (B) Vector map of p3XFLAG-ATS-BAP showing insertion of the phoA coding region into pFLAG-ATS-BAP. $\mu \mathrm{g} / \mathrm{mL}$ gentamycin. Cells were grown at $37^{\circ} \mathrm{C}$ in a humidified $\mathrm{CO}_{2}$ incubator with $5 \% \mathrm{CO}_{2}$. Transfection of the p3XFLAGCMV7-BAP plasmid was accomplished using LIPOFECTA-

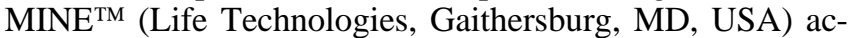
cording to manufacturer's directions. Two micrograms of vector DNA per plate were used for the transfection. Immunostaining was done $72 \mathrm{~h}$ after transfection.

\section{Immunostaining}

At $72 \mathrm{~h}$ after transfection, the cells were washed with 50 $\mathrm{mM}$ Tris- $\mathrm{HCl}, \mathrm{pH} 7.4,150 \mathrm{mM} \mathrm{NaCl}$ (TBS). The cells were fixed with 1:1 (vol/vol) methanol-acetone mixture for $1 \mathrm{~min}$. The fixed cells were washed $4 \times$ with TBS and then incubated with $10 \mu \mathrm{g} / \mathrm{mL} \mathrm{M} 2$ antibody-HRP conjugate in TBS for $1 \mathrm{~h}$. Cells were washed with TBS $5 \times$, and the M2 antibody-HRP conjugate was visualized with freshly prepared $0.01 \mathrm{mg} / \mathrm{mL}$ $o$-dianisidine, $0.015 \%$ hydrogen peroxide in TBS. Cells were stained for approximately $15 \mathrm{~min}$ and then visually inspected.

\section{RESULTS}

\section{Vector Construction}

We have constructed a vector for expression of proteins in mammalian host cells using a modified version of the FLAG expression system, which contains 3XFLAG sequences in tandem (Figure 1). This construct was designed to improve

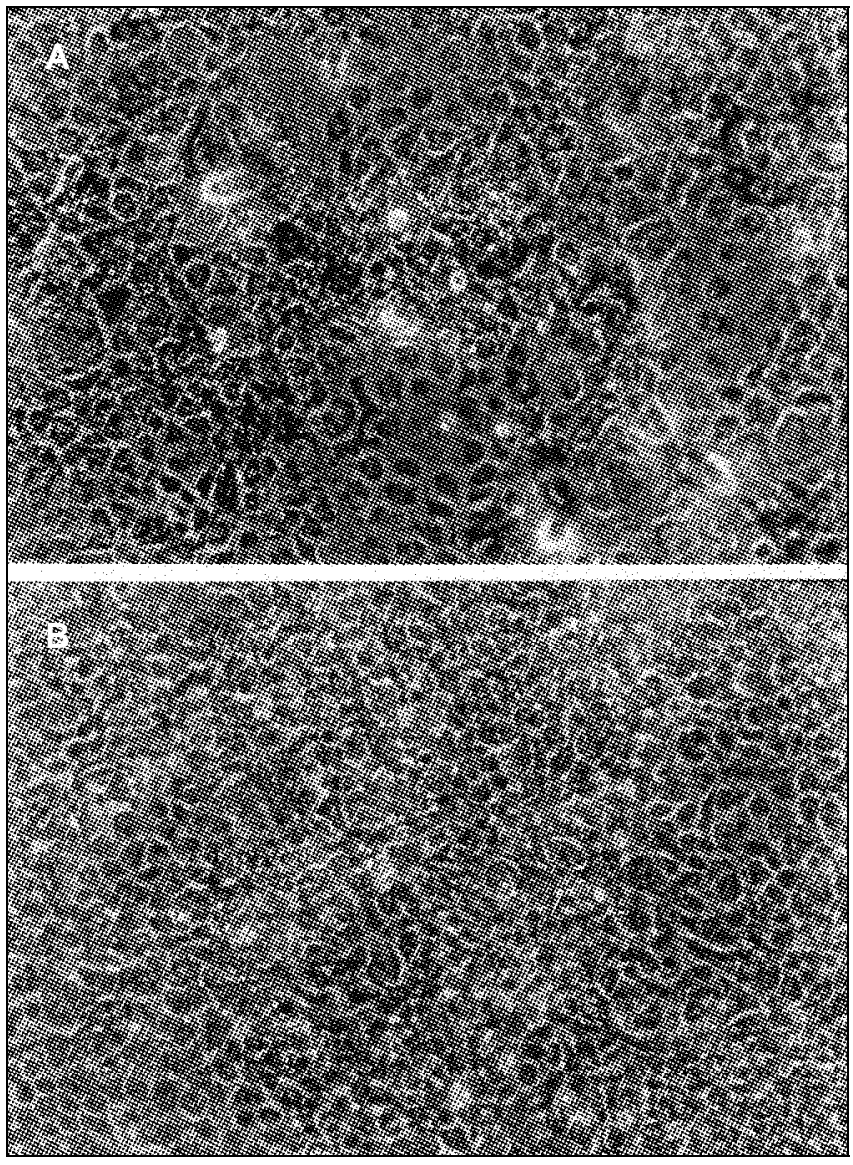

Figure 3. Light microscopy of immunostained cells. (A) COS-7 cells transfected with p3XFLAG-CMV-7-BAP. (B) Control COS-7 cells. 
the detection limit of expressed proteins in mammalian host cells. The first two flag peptides are modified FLAG sequences. The original FLAG epitope is Asp-Tyr-Lys-AspAsp-Asp-Asp-Lys, while the first two flag recognition sequences are Asp-Tyr-Lys-Asp-His-Asp with a Gly and a Ile spacer between the two sequences. These alternative sequences arise from phage display studies in which a different binding motif was determined (11). This allows the introduction of additional FLAG antibody binding sites without the addition of extra enterokinase recognition/cleavage sites.

The p3XFLAG-CMV-7 expression vector contains the promoter region of the human CMV major immediate early gene, which allows for constitutive expression of cloned genes in mammalian cell lines. The Kozak consensus sequence (8) is provided in the vector along with a multiple cloning site, which allows for a variety of cloning strategies. The multiple cloning site is compatible with the other existing CMV mammalian expression vectors. In addition, the expression vector contains the SV40 origin of replication for efficient high-level transient expression (12) and a DNA segment from the human growth hormone containing transcriptional termination sequence and polyadenylation signals (17). p3XFLAG-CMV-7 contains the $\beta$-lactamase gene for selection of the plasmid in E. coli.

\section{Expression of 3XFLAG-BAP in COS-7 Cells}

p3XFLAG-CMV-7-BAP (Figure 2) was transfected into
COS-7 cells as described in Materials and Methods. At $72 \mathrm{~h}$ after transfection, the cells were analyzed by immunostaining using an anti-FLAG M2-HRP conjugate. Figure 3 shows light microscopy of cells detected with M2 antibody and visualized with $o$-dianisidine.

\section{BAP Expression}

To address whether a triple FLAG fusion protein produces a more sensitive response then the traditional FLAG epitope, a triple FLAG version of BAP was constructed for expression in E. coli (Figure 2). The vector p3XFLAG-ATS-BAP was transformed into $E$. coli and the 3XFLAG-BAP expressed and purified as described in Materials and Methods. An N-FLAG-BAP containing the traditional epitope was also expressed and purified (3). A comparison of the sensitivity of the single and the triple FLAG-BAP was demonstrated by Western blot analysis as described above. Figure 4 shows the Western blot of purified single and triple FLAG that was probed with M2 antiFLAG antibody and detected by chemiluminescence. The results clearly indicate a tenfold increase in the detection limit of the triple FLAG-BAP as compared to the single FLAG-BAP fusion protein. We were able to detect $500 \mathrm{pg}$ of purified 3XFLAG-BAP with exposures as short as $1 \mathrm{~min}$. With increased exposure time, detection as low as $100 \mathrm{pg}$ has been achieved but with increased background (data not shown). We have also demonstrated a tenfold increase detection in both dot blot and ELISA (R. Hernan, personal communication). 


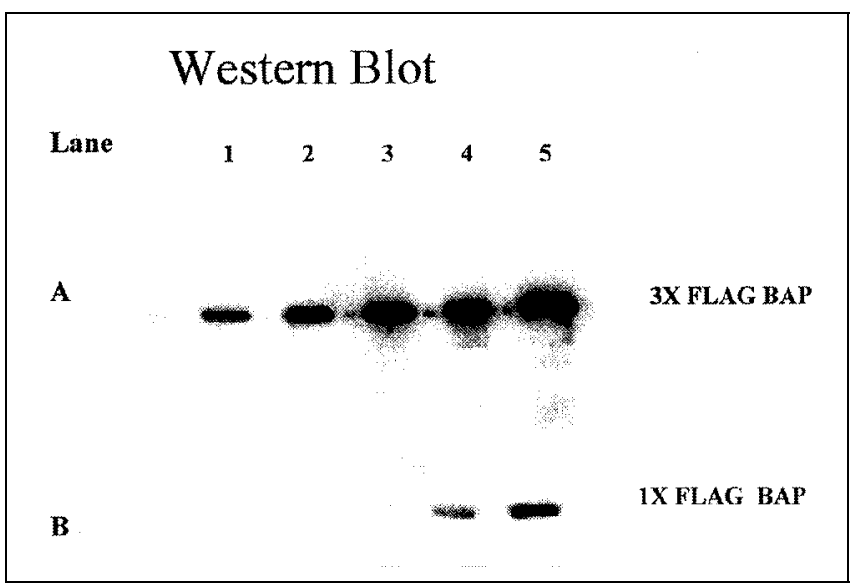

Figure 4. Detection of purified 3XFLAG-BAP by Western blot. (A) Western Blot of purified 3XFLAG-BAP using anti-FLAG M2 antibody. Lane 1, $0.5 \mathrm{ng}$; lane 2, $1 \mathrm{ng}$; lane 3, $2 \mathrm{ng}$; lane 4, $5 \mathrm{ng}$; and lane 5, $10 \mathrm{ng}$. (B) Western blot of purified N-FLAG-BAP using anti-FLAG M2 antibody. Lane 1, $0.5 \mathrm{ng}$; lane 2, $1 \mathrm{ng}$; lane 3, $2 \mathrm{ng}$; lane 4, $5 \mathrm{ng}$; and lane 5, $10 \mathrm{ng}$.

\section{DISCUSSION}

The FLAG epitope tag has been effectively used to detect and purify proteins (3) in mammalian and bacterial systems. We have demonstrated that the presence of three FLAG epitopes significantly increases the detection limit of purified BAP. Moreover, we have found that 3XFLAG-BAP cannot be eluted from anti-FLAG M2 affinity gel by competition with the original FLAG peptide. However, 3XFLAG-BAP and the 1XFLAG-BAP can be competitively eluted from the antiFLAG M2 affinity gel using 3XFLAG peptide (data not shown). The p3XFLAG-CMV-7 vector was designed for expression and detection of heterologous proteins in mammalian cells and is compatible with existing pFLAG-CMV vectors. This allows easy subcloning between vectors containing the single FLAG and the triple FLAG (Additional expression vectors encoding the triple FLAG sequence are in development). The immunostaining results show that expression of the phoA gene in COS-7 cells is not significantly perturbed by addition of the 3XFLAG sequence.

The M2 antibody reacts with the alternate epitope in the 3XFLAG sequence. In contrast, the M5 antibody fails to show the increased sensitivity of the M2 antibody (results not shown). Recent results using phage display (18) have demonstrated that the critical residues for M2 binding and M5 binding are slightly different. The M2 antibody prefers the sequence Asp-Tyr-Lys-Xxx-Xxx-Asp-Xxx-Xxx, while the M5 prefers Asp-Tyr-Xxx-Xxx-Asp-Asp-Xxx-Xxx. The triple FLAG sequence, Asp-Tyr-Lys-Asp-His-Asp, clearly favors the binding of M2 over that of the M5 or even the M1 antibody. This expression system allows for increased sensitivity and detection of the FLAG epitope tagging system with many similarities to the existing FLAG mammalian expression system.

\section{ACKNOWLEDGMENT}

The authors thank Richard Chubet and Fred Redeaux for their help in the beginning of this work, Hank Hwang for the M2-HRP conjugate and Kelly Prusheik for technical assistance and critical review of the manuscript.

\section{REFERENCES}

1.Andersson, S., H. Davis, H. Dahlbach, H. Jornvall and D. Russell. 1989. Cloning, structure and expression of the mitochondrial cytochrome P-450 sterol 26-hydroxylase, bile biosynthetic enzyme. J. Biol. Chem. 264:82228229 .

2.Brizzard, B. and R. Chubet. 1999. Epitope tagging of recombinant proteins, p. 5.8.1-5.8.11. In J.N. Crawley, C.R. Gerfen, R. McKay, M.A. Rogawski, D.R. Sibley and P. Skolnick (Eds.), Current Protocols in Neuroscience. John Wiley \& Sons, NY.

3.Brizzard, B., R. Chubet and D. Vizard. 1994. Immunoaffinity purification of FLAG epitope-tagged bacterial alkaline phosphatase using a novel monoclonal antibody and peptide elution. BioTechniques 16:730-735.

4.Dent, P., D. Reardon, D. Morrison and T. Sturgill. 1995. Regulation of raf- 1 and raf-1 mutants by ras dependent and ras independent mechanisms in vitro. Mol. Cell Biol. 15:4125-4135.

5.Evan, G., G. Lewis, G. Ramsay and J. Bishop. 1985. Isolation of monoclonal antibodies specific for human $\mathrm{C}$-myc proto-oncogene product. Mol. Cell Biol. 5:3610-3616

6.Hopp, T., V. Prickett, R. Price, R. Libby, D. March, D. Cerretti, D. Urdal and P. Conlon. 1988. A short polypeptide marker sequence useful for recombinant protein identification and purification. BioTechnology. 6:1204-1210.

7.Inouye, H., S. Michaelis, A. Wright and J. Beckwith. 1981. Cloning and restriction mapping of the alkaline phosphatase structural gene (phoA) of Escherichia coli and generation of deletion mutants in vitro. J. Bacteriol. 146:668-746.

8.Kozak, M. 1984. Point mutations close to the AUG initiator codon affect the efficiency of translation of rat preproinsulin in vivo. Nature 308:241.

9.Laemmli, V. 1970. Cleavage of the structural proteins during the assembly of the head of Bacteriophage T4. Nature 227:680-685.

10.Lee, J., J. Laydon, P. McDonnell, T. Gallagher, S. Kumar, D. Green, D. McNulty, M. Blumenthal et al. 1994. A protein kinase involved in the regulation of imflammatory cytokine biosynthesis. Nature 372:739-746.

11.Miceli, R., M. DeGraaf and H. Fischer. 1994. Two-stage selection of sequences from a random phage display library delineates both core residues and permitted structural range within an epitope. J. Immunol. Methods 167:279-287.

12.Okayama, H. and P. Berg. 1983. A cDNA cloning vector that permits expression of cDNA inserts in mammalian cells. Mol. Cell Biol. 3:280-289.

13.Overholt, S., T. Liu, D. Taylor, M. Wang, A.K. El-Naggar, E. Shillitoe, K. Adler-Storthz et al. 1997. Head and neck squamous cell growth suppression using adenovirus-p53-FLAG: a potential marker for gene therapy trials. Clin. Cancer Res. 3:185-191.

14.Prickett, K., D. Amberg and T. Hopp. 1989. A calcium dependent antibody for identification and purification of recombinant proteins. BioTechniques 7:580-589.

15.Ritchie, P., A. Decout, J. Amey, C. Mann, J. Read, M. Rosseneu, J. Scott and C. Shoulders. 1999. Baculovirus expression and biochemical characterization of the human microsomal triglyceride transfer protein. Biochem. J. 338:305-310.

16.Schulte am Esch, II, J., J. Sevigny, E. Kaczmarek, J. Siegel, M. Imai, K. Koziak, A. Beaudoin and S. Robson. 1999. Structural elements and limited proteolysis of CD39 influence ATP diphosphohydrolase activity. Biochemistry 38:2248-2258.

17.Seeburg, P. 1982. The human growth hormone gene family: nucleotide sequences show recent divergence and predict a new polypeptide hormone. DNA 1:239-249.

18.Slootstra, J., D. Kuperus, A. Pluckthun and R. Meloen. 1996. Identification of new tag sequences with differential and selective recognition properties for the anti-FLAG monoclonal M1, M2 and M5. Mol. Divers. 2:156-164.

19.Thomsen, D., R. Stenberg, W. Goins and M. Stinski. 1984. Promoterregulatory region of the major immediate early gene of human cytomegalovirus. Proc. Natl. Acad. Sci. USA 81:659-663.

20.Xu, T. and G. Rubin. 1993. Analysis of genetic mosaics in developing and adult Drosophila tissues. Development 117:1223-1237.

Address correspondence to Bill Brizzard, Sigma Chemical Company, 3050 Spruce Street, St. Louis, MO 63103, USA. Internet:bbrizzard@sial.com 